\title{
Binocular summation in the perception of form at brief durations'
}

\author{
CHARLES W. ERIKSEN, THOMAS S. GREENSPON, JOSEPH LAPPIN, UNIVERSITY OF ILLINOIS \\ WILLIAM A. CARLSON, DANVILLE VA HOSPITAL
}

Visual form identification at brief durations was studied under: (a) wonocular presentation; (b) dichopic presentation where the same form was presented successively on noncorresponding areas; and $(c)$ dichopic presentation where the same form was presented on corresponding areas simultaneously and successively. Form identification for noncorresponding area dichopic presentation was at the level to be expected from 2 independent chances to perceive. Both simultaneous and successive dichopic presentation on corresponding areas gave identification accuracy significantly above the level predicted by the assumption of independence. However, the binocular summation was not complete. When the same amount of energy entering the visual system in a binocular presentation was given in a monocular stimulation, the latter condition gave significantly better identification.

The superiority of binocular viewing in a detection situation seems well established (Collier, 1954; Matin, 1962; Pirenne, 1943; Shaad, 1935; Wolf \& Zigler, 1963) although the basis for this superiority is still a matter of controversy. Some view binocular vision as essentially two monocular systems working together. From this viewpoint the increased sensitivity in brightness thresholds that occurs with binocular regard can be attributed to the gain accruing from two independent chances to detect the target. Pirenne (1943) seems to have been the first to apply an independence probability model to binocular threshold data, and he concluded that the reduction in binocular over monocular thresholds was no greater than could be attributed to two independent detectors.

More recent work by Collier (1954) and Matin (1962) has shown that the gain in binocular detection is greater than can be attributed to independent detectors. Instead, their data suggest a summative process from the two eyes. Levelt (1965), a recent exponent of an interactionist or summative theory of binocular vision, has advanced a theory of brightness interaction in which total brightness depends upon a weighted average of the luminance contributions from each eye where the weights add to a constant. Further, the weighting assigned to the input from each eye is in part dependent upon contour information provided to that eye.

The present experiment is concerned with the contribution of binocular vision to form identification at brief durations and low luminance. Nearly all of the experimental work on interocular summation has been concerned with brightness but there is reason to expect that a study of the interaction or possible summative role of the two eyes in form identification might yield different results as well as extend our general knowledge of binocular vision. Levelt's (1965) as well as Bouman's (1955) work has suggested that contour information is processed differently in binocular vision than is brightness and an assessment of the effects of binocular vision on form identification would seem to be a necessary step in the process of understanding such binocular phenomena as rivalry and suppression.

\section{Method}

Subjects. Six Ss, four males, served as paid volunteers. They were selected on a basis of their having essentially similar form identification functions in each of their eyes. They also represented a relatively homogeneous group in terms of exposure duration necessary to obtain above-chance identification accuracy for the three forms employed.

Apparatus. Adichopic viewing system was constructed and was used along with programmer, timers and lamp driver from a Scientific Prototype Model GA threefield tachistoscope. The optical system is shown schematically in Fig. 1. Stimuli mounted on white plastic cards could be presented in the stimulus card holders in the right and left eye stimulus fields and were frontlighted by two Sylvania F-4G5/CWX bulbs which in turn were pulsed and programmed by the Scientific Prototype equipment. A light-proof baffle (B) obscured all but a circle of $2^{\circ}$ of angle diameter of the stimulus display. This circular field was reflected off front surface mirrors (M) back through a beamsplitting mirror (BS) and then through tubes made of black construction paper which terminated at the viewing hood.

The $S$ rested his head on a hood visor to help maintain a constant position and viewed the displays through the black tubes. The diameter of these tubes was such that the ends of the tubes exactly coincided with the $2^{\circ}$ diameter circular holes in the baffles in the stimulus compartments. Luminance from the adapting field, diffused through milk glass, was reflected from the beam splitter (BS) to the S's eye. Fixation for both the right and left eye fields was a backlighted " $\mathrm{X}, " .12^{\circ}$ of angle, which was obtained by a solid black card positioned immediately behind the stimulus card but having the "X" cut out of it. Light for the "X" was provided by GE 44 bulbs in the back of the stimulus compartments. The " $X$ " was visible to the $S$ with a stimulus card in place by means of a circular hole cut in the center of the stimulus card that positioned over the luminous 


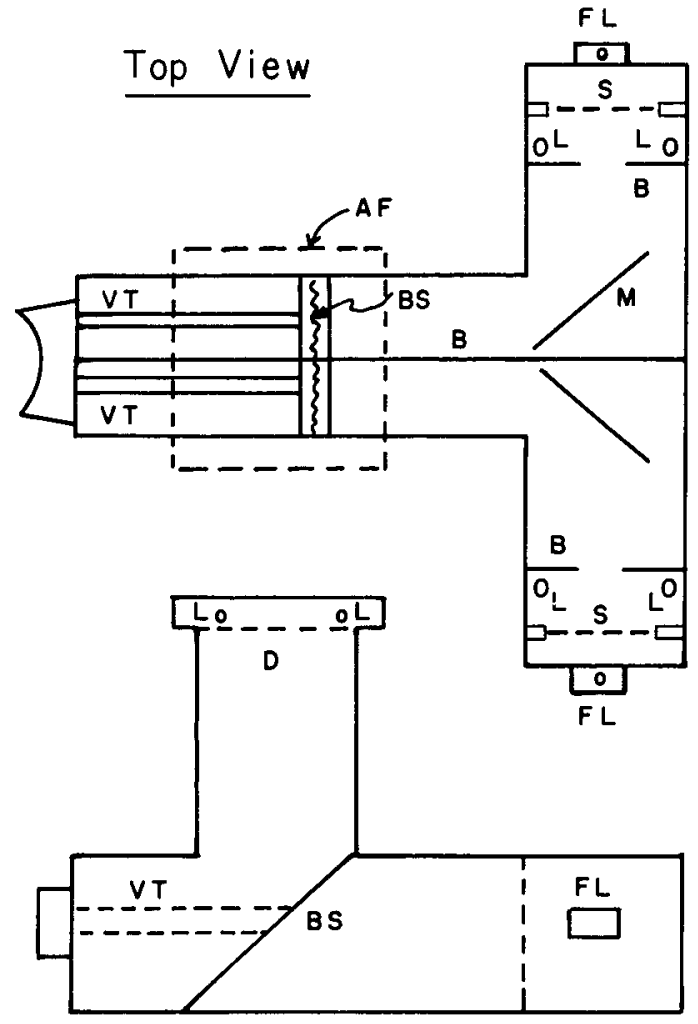

\section{Side View}

Fig. 1. Schematic top and side views of the dichopic viewing system. The major parts and construction are as follows: (a) FL fixation light sources; (b) $\mathrm{S}$ - stimulus holders; (c) L - Sylvania F4 T5/CWX lamps; (d) B - light proof baffles; (e) $M$ - front surface mirrors; (f) BS - beam splitter; (g) AF - adaptation field; and (h) VT - viewing tunnels.

"X." The luminance of the fixation point was such that it was clearly visible against the background adapting field luminance.

The stimuli were the capital letters A, T and U, obtained from Paratype No. 11316, 18-point Futura bold and subtended $.12^{\circ}$ of visual angle. They occurred at the corners of an imaginary $1^{\circ}$ square centered on the " $X$ " fixation point. The letters were mounted on white vinyl cards and had approximately $96 \%$ contrast. The fixation point was on constantly. Both stimulus fields and the adapting field had a constant luminance of $.3 \mathrm{~mL}$.

Procedure. Before participating in the experimental sessions each $\mathrm{S}$ was given two practice sessions during which he identified the three letters presented under dichopic conditions. Here the same letter was presented to the two eyes on corresponding retinal areas simultaneously. During the last practice session an exposure duration was determined for each $\mathrm{S}$ that yielded 70 to
$75 \%$ identification accuracy. This duration is referred to hereafter as time $T$. All Ss achieved the criterion at exposure durations in the range of 20 to $25 \mathrm{msec}$. (mean $=23$ ).

Following the practice sessions each S served for one session on each of six experimental days. During each session $S$ received four blocks of 24 trials except for the last session where only two blocks of trials were run. There were 11 experimental conditions that were randomly distributed into the available blocks. The 11 conditions were: (a) stimulus presented to the right eye alone at time $T$; (b) left eye alone at time $T$; (c) right eye at time $2 \mathrm{~T}$ (twice the exposure duration for the $\mathrm{S}$ that was used in conditions a and b); (d) left eye at time 2T; (e) right and left eye simultaneously on corresponding retinal areas at time. $T$; (f) right eye followed immediately by stimulation to left eye, each eye for time $\mathrm{T}$; (g) left eye followed immediately by stimulation to right eye on correspoding areas, each eye for time $\mathrm{T}$; (h) right eye followed immediately by left eye stimulation each for time $\mathrm{T}$ on noncorresponding heterolateral retinal areas; (i) left eye followed immediately by stimulation to right eye each for time $\mathrm{T}$ on noncorresponding heterolateral areas; (j) right eye followed by left eye on noncorresponding homolateral areas; and (k) left eye followed immediately by right eye on noncorresponding homolateral areas.

The stimuli appeared randomly in the four corners of the imaginary square except for the noncorresponding homo- and heterolateral conditions. For the unfused homolateral conditions the two occurrences of the letter on a trial were on the same side of the square, one in the upper corner and one in the lower corner. For the heterolateral unfused condition the two occurrences of the letter were either on the two bottom corners or the two upper corners.

In order to control for luminance summation-contrast reduction effects (Eriksen, 1966a) during the conditions of successive stimulation, a letter was exposed to one eye while the other eye remained dark, then the reverse occurred. The adapting field remained off during the entire stimulation sequence. For single eye stimulation one eye was presented a letter while the other eye received darkness; then the other eye received a blank illuminated field while the first eye received a dark period. For the fused simultaneous condition both eyes were simultaneously stimulated with the letter form and followed by a period of darkness equal to the exposure duration before return of the adapting field luminance. For the unfused conditions the sequence of stimulation was the same as for the monocular conditions.

Before each trial, on a ready signal from $E, S$ fixated the " $\mathrm{X}$ " in each field and when they were fused and the " $\mathrm{X}$ " appeared sharp and clear, he triggered a stimulation. Upon completion of the stimulation the $\mathrm{S}$ gave a forced-choice response from among the three possible letters along with a confidence rating as to his certainty 
Table 1. Percent Correct Form Identification by Ss and Experimental Viewing Conditions

\begin{tabular}{|c|c|c|c|c|c|c|c|c|}
\hline \multirow[b]{3}{*}{ Subjects } & \multicolumn{4}{|c|}{ Monocular } & \multicolumn{4}{|c|}{ Dichopic } \\
\hline & \multicolumn{2}{|c|}{ Right eye } & \multicolumn{2}{|c|}{ Left eye } & \multicolumn{2}{|c|}{ Fused } & \multicolumn{2}{|c|}{ Unfused } \\
\hline & Time T & Time $2 \mathrm{~T}$ & Time $T$ & Time $2 T$ & Simultaneous & Successive & Homolateral & Heterolateral \\
\hline$M N$ & 38 & 83 & 46 & 79 & 63 & 69 & 62 & 59 \\
\hline SH & 58 & 75 & 44 & 36 & 69 & 70 & 66 & 66 \\
\hline$J M$ & 54 & 98 & 43 & 92 & 79 & 81 & 59 & 69 \\
\hline $\mathrm{CE}$ & 44 & 96 & 57 & 98 & 78 & 86 & 68 & 58 \\
\hline $\mathrm{JC}$ & 71 & 92 & 46 & 88 & 71 & 82 & 60 & 63 \\
\hline WL & 48 & 92 & 73 & 94 & 75 & 74 & 65 & 72 \\
\hline & 313 & 536 & 309 & 547 & 435 & 462 & 380 & 387 \\
\hline Mean & 52 & 89 & 52 & 91 & 73 & 77 & 63 & 64 \\
\hline
\end{tabular}

of his judgment. Numbers 1,2 and 3 were used to reflect his confidence with 1 indicating very certain and 3 a guess.

\section{Results and Discussion}

The data were first analyzed for a possible sequential order effect of right eye to left eye or left eye to right eye in the three conditions involving successive stimulation under dichopic conditions. The percent correct form identifications under fused successive, unfused homolateral and unfused heterolateral were analyzed in a three-way classification analysis of variance (conditions, right-left or left-right eye sequence, and Ss). The only significant effect was that attributable to conditions $(F=8.7, \mathrm{df}=2 / 10, \mathrm{p}<.01)$ which reflected a superiority of the fused condition over the two unfused conditions. The lack of a significant Seffect was anticipated since the exposure duration for each $\mathrm{S}$ had been adjusted to yield essentially comparable performance over Ss on the basis of the $S^{\prime}$ 's performance during the practice sessions. The sequence order variable and its interactions gave $\mathbf{F}$ ratios of less than 1 .

In Table 1 the percent correct forced-choice form identifications are shown by Ss for each of the eight main experimental conditions. Average values are also shown in Table 1. In view of the lack of significant or suggestive effects attributable to right-left or left-right stimulation sequence, the data obtained under these two sequences of stimulation for the fused successive and the unfused homo- and heterolateral conditions have been pooled.

of major interest in Table 1 is the percent correct form identifications under the two fused and the two unfused dichopic conditions. As is seen, both the successive and the simultaneous fused conditions are superior to either of the unfused conditions. The significance of the differences was evaluated by a two-way classification analysis of variance (conditions and Ss). The differences between the four conditions were significant beyond the .01 level $(F=9.6, d f=3 / 15)$. None of the other effects approached significance. Comparison of the four condition means with each other revealed that the fused successive and the fused simultaneous conditions differed from both the unfused conditions atbeyond the .01 level. There was no significant difference between the two unfused conditions, but there is a suggestion that for fused dichopic stimulation successive is superior to simultaneous. However, this difference did notattain significance $(p>.10)$.

While presenting the same form either simultaneously or successively on corresponding retinal areas is superior to presentation to noncorresponding areas, the latter conditions show considerable gain in identification over that obtained by presentation to either eye alone. It is tempting to conclude that the performance under stimulation of corresponding retinal areas for the fused conditions represents a binoculàr summation process whereas the stimulation to noncorresponding or unfused areas represents the gain over monocular stimulation attributable to two chances to perceive or identify the form. However, other possibilities must be considered. Performance under the unfused dichopic conditions might also represent a binocular interaction. The obtained level of performance could represent some summative process which is not as great as when the dichopic stimulation is on fused areas. Alternatively, it is possible that the poorer performance for the unfused relative to the fused conditions may represent an inhibitory process operating interocularly for stimuli that do not fall on corresponding points. This latter alternative would require an extremely short latency for the inhibitory process since stimulus durations were on the order of 20 to $25 \mathrm{msec}^{2}$

Interpretation becomes clearer if we can compute or determine what the level of performance would be if the two eyes behaved as independent perceptual units. Previous models, in attempting to compute the level of performance to be expected from independent chances, have followed Pirenne (1943) which is unfortunate since Pirenne made a serious error which overestimates the level of performance to be expected on the assumption of independence. He used the formula

$$
\text { P1 + P2 - P1 P2 }
$$

where P1 and P2 refer to the probabilities of detection in the separate eyes under monocular stimulation. This formula is correct when applied to such events as coins or dice, but psychophysical detections or hit rates involve a guessing component, particularly if a forcedchoice procedure is employed. Thus, the percent correct form identification in the present experiment for left 
eye stimulation represents not only the number of times $S$ perceived the form but also the number of times he guessed correctly when he did not perceive it.

The problem involved in computing the level of performance to be expected with independent chances has been discussed elsewhere (Eriksen, 1966b) along with models that provide a more appropriate prediction based on the independence assumption. $3 \mathrm{~A}$ close approximation to the level to be expected from independent systems can be obtained by correcting the monocular hit rates for guessing. Let $\mathrm{P}_{R}$ and $\mathrm{P}_{\mathrm{L}}$ be the monocular hit rates for the right and left eyes respectively. Then $1-P_{L}$ is the percent of incorrect responses for the left eye. With an a priori probability of a correct guess being $1 / 3$, then $3 / 2$ of $1-P_{R}$ and $3 / 2$ of $1-P_{L}$ would lead to an estimate of the total percent of the time that $S$ guessed for the respective monocular presentations. If we let $G_{R}$ and $G_{L}$ represent the total percent of guess trials for monocular presentations to the respective eyes, then for a dichopic presentation $1-G_{R} G_{L}$ is the percent of times the $S$ would correctly perceive the form with one eye or the other or both. This value must then have added to it the number of correct guesses that occur on the occasions when $S$ fails to perceive the form with either eye. Thus the percent correct form identifications to be expected if the eyes behaved as independent systems is given by

$$
1-G_{R} G_{L}+1 / 3 G_{R} G_{L}
$$

This two-state model was applied to the data of the present experiment. Each S's monocular hit rates were used to compute what the hit rate should be based on two independent chances to perceive. These predicted values were then averaged through the six Ss and gave a predicted hit rate of $66 \%$. This value agrees very closely with the values of 63 and $64 \%$ obtained under the two unfused dichopic conditions. As expected, the direction of error is toward overestimation.

The close agreement between the hit rates for the unfused dichopic conditions and the value predicted from the model for independence are strongly suggestive that the gain in the nonfused conditions over monocular stimulation represents what could be expected from two independent opportunities to perceive the stimulus. The superiority of the fused over the unfused dichopic conditions then represents an actual interocular summation process. This conclusion is reinforced by the fact that the above model for independence has been shown in several other perceptual situations to produce close predictions of the effects of independent opportunities to perceive (Eriksen, 1966b; Eriksen \& Lappin, 1965; Eriksen \& Lappin, in press).

While there seems to be a genuine summative effect in form identification, the summation does not appear to be complete. This can be seen by comparing the dichopic fused conditions with the monocular time $2 \mathrm{~T}$ conditions. In these four conditions the energy entering the visual system is the same and in the case of the dichopic fused successive condition the energy is distributed over the same time span as for the monocular $2 \mathrm{~T}$ conditions. (The time $2 \mathrm{~T}$ conditions are monocular stimulations at twice the exposure durations of the time $T$ conditions.) For all Ss monocular stimulation in either eye at time $2 \mathrm{~T}$ yields appreciably greater form identification than that obtained for the dichopic fused conditions. This failure to obtain complete dichopic summation might be attributed to inertia-like effects involved in the activation of the individual sensory receptor cells in the separate eyes. It also may reflect a basic contour development time such as reported by Kahneman (1965) which has to be duplicated in the separate eyes in the case of dichopic stimulation. There is also the possibility of uncorrelated nystagmic movements in the two eyes that lead to occasional trials on which the stimulations do not fall exactly on corresponding areas. This latter possibility could be ruled out using stabilized image techniques.

It is apparent from the data that there is no difference for the dichopic unfused stimulation whether the stimuli falling on noncorresponding areas follow homolateral or heterolateral pathways. If the two stimulations fall in the nasal retina of one eye and the temporal retina of the other both stimulations go to the same brain hemisphere as opposed to the case where both stimulations fall on the nasal or on the temporal retinas in the separate eyes. One might have anticipated a difference in these two methods of unfused stimulation on the basis of correlated sensitivity or alternatively, "noise" within the separate hemispheres. Failure to find a difference between homo- and heterolateral stimulation suggests that the noise or sensitivity is as uncorrelated within a hemisphere as across hemispheres. This is consistent with previous findings that have indicated that for stimulations in the fovea at least a degree or more apart, perceptual system error is uncorrelated at a given moment in time (Eriksen, 1966a; Eriksen, Munsinger, \& Greenspon, 1966; E riksen \& Lappin, in press).

\section{References}

Barlow, H. B., FitzHugh, R., \& Kuffler, S. W. Change of organization in the receptive fields of the cat's retina during dark adaptation. J. Physiol., 1957, 137, 338-354.

Bouman, M. A. On foveal and peripheral interaction in binocular vision. Optica Acta, 1955, 1, 177-183.

Collier, G. Probability of response and intertrial association as functions of monocular and binocular stimulation. J. exp. Psychol., 1954, 47, 75-83.

Eriksen, C. W. Temporal luminance summation effects in backward and forward masking. Percept. \& Psychophys., 1966a, 1, 87-92.

Eriksen, C. W, Independence of successive inputs and uncorrelated error in visual form perception. J. exp. Psychol., 1966b, 72, 26-35.

Eriksen, C. W., \& Lappin, J. S. Internal perceptual system noise and redundancy in silumtaneous inputs in form identification. Psychon. Sci., 1965, 2, 351-352.

Eriksen, C. W., \& Lappin, J. S. Independence in the perception of simultaneously presented forms at brief durations. J. exp. Psy- 
chol., in press.

Eriksen, C. W., Munsinger, H. L., \& Greenspon, T. S. Identification versus same-different judgment: An interpretation in terms of uncorrelated perceptual error. J.exp. Psychol., 1966, 72 , 20-25.

Kahneman, D. Exposure duration and effective figure-ground contrast. Quart. J. exp. Psychol., 1965, 17, 308-314.

Levelt, W. J. M. Binocular brightness averaging and contour in formation. Brit. J. Psychol., 1965, 56, 1-13.

Matin, L. Binocular summation at the absolute threshold of peripheral vision. J. Opt. Soc. Amer., 1962, 52, 1276-1286.

Pirenne. M. H. Binocular and uniocular thresholds of vision. Nature, $1943 ; 48,43-63$.

Shaad, Dorothy. Binocular summation in scotopic vision. $J$. exp. Psychol, 1935, 4, 391-413.

Wolf, E., \& Zigler, M. J. Effects of uniocular and binocular excitation of the peripheral retina with test fields of various shapes on binocular summation. J. Opt. Soc. Amer., 1963, 53, 1199-1205.

\section{Notes}

1. This investigation was supported by Public Health Service Research Grant MH-1206 and a Public Health Service Research Career Program Award K6-MH-22014. The authors wish to acknowledge their appreciation to Roy M. Hamlin, Research Psychologist, and other members of the staff at the Danville Veterans Administration Hospital where pilot studies were carried out for this experiment.

2. There is some evidence that such short latencies of inhibition are not probable, even within one eye, Barlow, FitzHugh and Kuffler (1957) present such evidence as an incidental finding in a study on the relationship of Ricco's law within one retinal ganglion cell receptive field of the cat. As the area of a circular stimulus to an RF increases, threshold for firing of the ganglion cell drops, until a critical diameter is reached. At this point the threshold rises again due to the inclusion of an inhibitory annulus in the stimulated area. This relationship was true for stimulus flashes of $380 \mathrm{msec}$. but did not hold when the flash was only $7 \mathrm{msec}$; in this case the threshold tended toward an asymptote. The authors ascribe this to either a differential latency for inhibition and excitation or to a kind of inertia effect in which inhibition must build up over a longer period of time. With the added complication that central mechanisms involve, it can be seen that interocular inhibition probably has a reasonably long latency.

3 . The assumption that there are only two kinds of trials, those where $S$ perceives and those where he guesses, is an oversimplification. Eriksen (1966b) has presented a more elaborate model based upon several states of partial information in the $\mathbf{S}$ following a stimulus presentation. These states are estimated via the S's confidence rating associated with each judgment. A two-state model such as described above will tend to overestimate the hit rate for two independent chances to perceive if the number of different information states existing in the $S$ is greater than two. Confidence judgments were obtained from $S s$ in the present experiment but an error was made in allowing the $\mathrm{Ss}$ to distribute the confidence range over the entire range of experimental conditions. As a consequence the Ss used their high confidence judgments for the fused and unfused dichopic conditions where more information was obtained and a higher confidence existed. Judgments on the monocular conditions were almost invariably given a confidence rating of "cuess."

(Accepted for publication October 5, 1966.) 